\title{
əobserving Polar Regions from Space: Comparison between Highly Elliptical Orbit and Medium Earth Orbit Constellations
}

\author{
AlEXANDER P. TRISHCHENKO \\ Canada Centre for Remote Sensing, Natural Resources Canada, Ottawa, Ontario, Canada \\ LOUIS GARAND \\ Meteorological Research, Environment and Climate Change Canada, Dorval, Quebec, Canada \\ LARISA D. TRICHTCHENKO \\ Canadian Space Weather Forecast Center, Natural Resources Canada, Ottawa, Ontario, Canada
}

(Manuscript received 22 February 2019, in final form 7 June 2019)

\begin{abstract}
Continuous observation of polar regions from space remains an important unsolved technical challenge of great interest for the international meteorological community. This capacity would allow achieving global continuous coverage once combined with the geostationary (GEO) satellite network. From a practical point of view, continuous coverage of polar regions with a small number of spacecraft can be obtained from a constellation of satellites either in highly elliptical orbits (HEO) or in medium Earth orbits (MEO). The study compares HEO and MEO satellite constellations for their capacity to provide continuous imaging of polar regions as function of the viewing zenith angle (VZA) and evaluates the corresponding latitude limits that ensure sufficient overlap with GEO imagery. Earlier studies assumed the latitude boundary of $60^{\circ}$ and the VZA range $70^{\circ}-85^{\circ}$ depending on the space mission focus: meteorological purposes or communications. From the detailed analysis of meteorological retrieval requirements, this study suggests that the overlap of the GEO and polar observing systems (HEO or MEO) should occur down to the latitude band $45^{\circ}-50^{\circ}$ with a maximum VZA ranging between $60^{\circ}$ and $64^{\circ}$. This coverage requirement can be met with two sets of three-satellite HEO constellations (one for each polar area) or a six-satellite MEO constellation. The 12-h Molniya and 14-, 15-, and 16-h HEO systems have been analyzed and determined to meet these revised requirements. The study demonstrates that the six-satellite 24-h MEO system can provide a suitable solution, which is also beneficial from the point of view of ionizing radiation and image acquisition geometry. Among the HEO systems, the 16-h HEO has some advantages relative to other HEO systems from the point of view of spatial coverage and space radiation.
\end{abstract}

\section{Introduction}

Satellite meteorology has achieved tremendous progress since the launch of the first successful meteorological low Earth orbit (LEO; appendix B gives the definitions for many of the acronyms and variables used in this paper) satellite TIROS-1 in 1960 and the beginning of meteorological imaging from geostationary

Denotes content that is immediately available upon publication as open access.

Corresponding author: Alexander Trishchenko, alexander. trichtchenko@canada.ca
(GEO) orbit in 1974. Nevertheless, a satellite meteorologist's dream to observe weather at any point and time over the globe remains unaccomplished. From the orbital point of view, the practical solution for continuous imaging capacity may rely on two concepts: 1 ) the highly elliptical orbit (HEO) satellite constellation or 2) the medium Earth orbit (MEO) satellite constellation. To achieve continuous global coverage, these systems should complement the GEO satellite network that is currently operated by a number of countries. The implementation of LEO solution for this purpose is not practical because it would require a large number of satellites (Trishchenko and Garand 2012). 

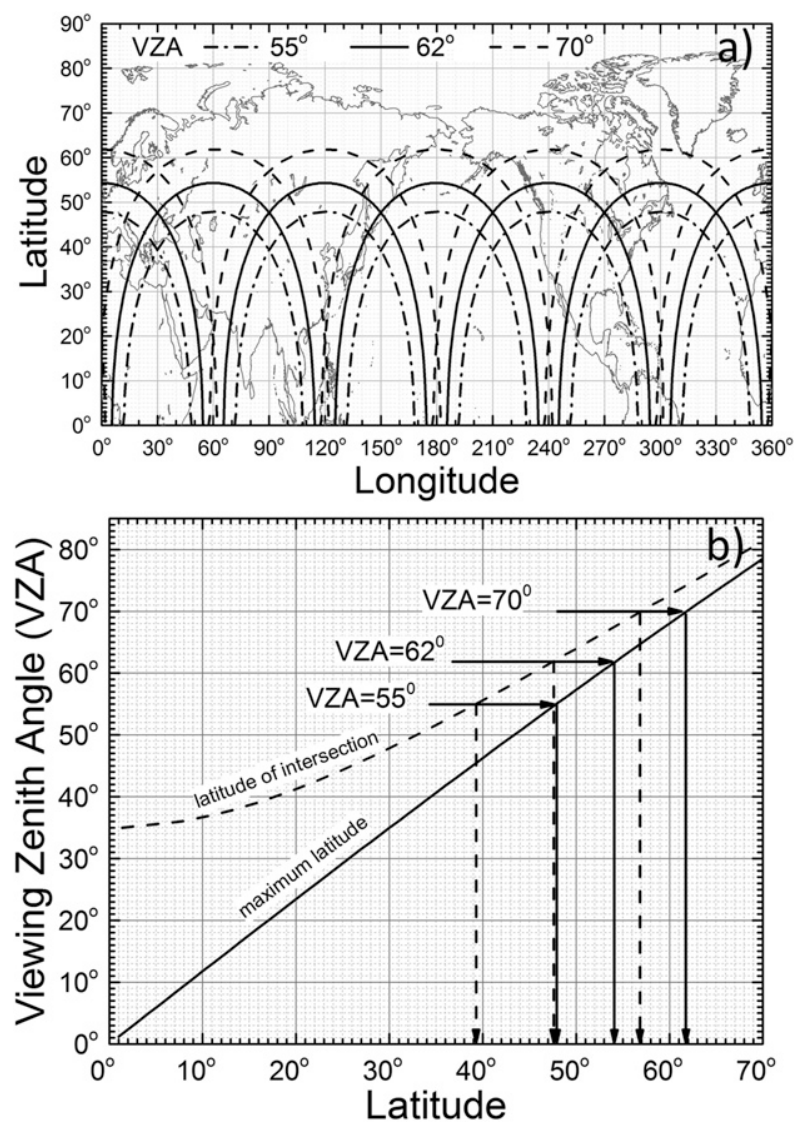

FIG. 1. (a) Map of spatial coverage from the network of GEO satellites, and (b) the VZA-vs-latitude relationship for GEO imaging.

To visualize the issue, Fig. 1a displays the spatial coverage of the Northern Hemisphere from a constellation of six GEO satellites equally spaced over the equator. Although the real currently operational GEO constellation is configured differently, the illustration provides a very good approximation of the service area covered by the entire operational GEO constellation. Figure $1 \mathrm{~b}$ shows the maximum observed latitude and maximum latitude of the field-of-view (FOV) intersection between two adjacent GEO satellites for various limits of the viewing zenith angle (VZA): $55^{\circ}, 62^{\circ}$, and $70^{\circ}$, which can be considered as representative of low, medium and high VZA limits employed in retrievals, as discussed later on. Detailed viewing geometry, definition of VZA and other angles utilized in this study are discussed in appendix A (and displayed in Fig. A1 included there). One can see that latitude $60^{\circ}$ provides an approximate lower boundary that needs to be covered by HEO or MEO constellation to complement the coverage available from GEO satellites, assuming a VZA limit of $70^{\circ}$.

The World Meteorological Organization (WMO) identified the need for HEO-based satellite observing

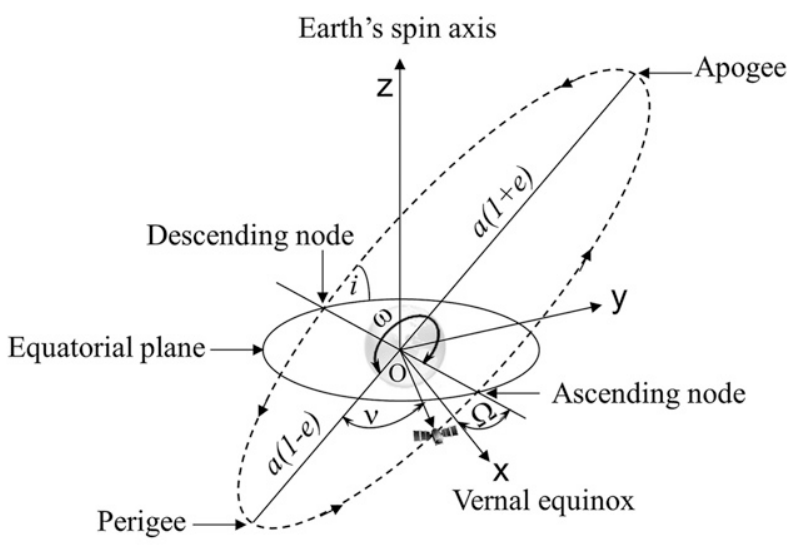

$\mathrm{O}$ - center of the Earth
$a$ - semi-major axis
$e$ - eccentricity
$i$ - orbit inclination angle
$v$ - mean anomaly angle (MAA)
$\omega$ - argument of perigee
$\Omega$-right ascension of ascending node (RAAN)

FIG. 2. Graphical layout of elliptical orbit and definition of Keplerian orbital elements.

system in its Vision 2025 document and in the new Vision 2040 strategic plan to be adopted in 2019 (WMO 2009, 2017). The HEO concept for meteorological imaging has been introduced by Kidder and Vonder Haar (1990) and analyzed in a number of studies. The Russian Arktika-M space mission on 12-h Molniya orbit is scheduled for launch in late 2020 (WMO 2019). The benefits of HEO observations for global weather forecasting has been demonstrated by Garand et al. (2013).

It is beneficial for the HEO constellation to be in the orbital plane or planes with critical inclination equal to $63.435^{\circ}$ that minimizes orbit maintenance related to the drift of the argument of perigee $\omega$ (see Fig. 2 for definition of orbital elements). This drift causes the repositioning of apogee to lower latitude with time and therefore reduces the coverage of high-latitude regions. Trishchenko and Garand (2011) provided a detailed analysis of 12-h Molniya HEO system and showed that continuous imaging of the Northern polar region can be achieved above $\sim 58^{\circ} \mathrm{N}$ from two satellites with the VZA limit of $70^{\circ}$. Two HEO constellations are required, one for each hemisphere (Northern and Southern). A twosatellite constellation in one orbital plane is preferred for data assimilation in the global weather forecast system because of the more uniform spatial distribution of viewing conditions over the polar region. The imaging time should be at least $16 \mathrm{~h}$ per day for each satellite (equally split before and after the apogee point) to ensure nearly continuous sampling of the polar region 
TABLE 1. Key values of latitude for GEO FOV as function of VZA.

\begin{tabular}{lccl}
\hline \hline VZA & Max FOV lat & Lat of FOV intersection & \multicolumn{1}{c}{ Reference for VZA retrieval limit } \\
\hline $55^{\circ}$ & $47.9^{\circ}$ & $39.3^{\circ}$ & NOAA (2016) \\
$60^{\circ}$ & $52.5^{\circ}$ & $45.3^{\circ}$ & NOAA (2016) \\
$62^{\circ}$ & $54.3^{\circ}$ & $47.7^{\circ}$ & NOAA (2016) \\
$65^{\circ}$ & $57.1^{\circ}$ & $51.2^{\circ}$ & NOAA (2016), Kazuki (2017), Takahito and Daisaku (2016) \\
$68^{\circ}$ & $60.0^{\circ}$ & $54.7^{\circ}$ & Hernandez-Carrascal and Bormann (2014) \\
$70^{\circ}$ & $61.8^{\circ}$ & $57.0^{\circ}$ & NOAA (2016), Daisaku (2016) \\
$73.3^{\circ}$ & $65.0^{\circ}$ & $60.8^{\circ}$ & EUMETSAT (2011) \\
\hline
\end{tabular}

(scenes available every $10 \mathrm{~min}$ or less). Trishchenko et al. (2011) proposed the three apogee (TAP) 16-h HEO system to reduce the hazardous ionizing radiation dose from high-energy protons. The two-satellite TAP HEO constellation offers an attractive orbital solution with three stable apogees separated by $120^{\circ}$ in latitude. A comprehensive analysis of ionizing space radiation conditions for the HEO systems with orbital periods ranging from 6 to $24 \mathrm{~h}$ has been presented in Trichtchenko et al. (2014). Based on this analysis, Trishchenko et al. (2016) introduced the multipleapogee (MAP) HEO systems, notably with periods of 14 and $15 \mathrm{~h}$, that have advantage of more uniform distribution of viewing conditions over the polar zone, which is beneficial for climate and weather applications. Some additional optimization of orbital parameters to further reduce the total ionizing dose for the 14-, 15-, and 16-h HEO systems has been discussed in Trishchenko et al. (2019). A two-satellite HEO constellation named Atmospheric Imaging Mission for Northern Regions (AIM-North) has been proposed for air quality and green gas monitoring (Nassar et al. 2019). It is understood from these previous studies that several variants of two-satellite HEO systems launched at the critical inclination $63.435^{\circ}$ can achieve continuous coverage of the polar latitude zone starting from about $58^{\circ}$ to $60^{\circ}$ at the VZA limit of $70^{\circ}$.

The MEO is broadly defined as an orbit with altitude that is too high to be classified as LEO and does not belong to the HEO or GEO types. Chobotov (2002) applies the MEO category to any small eccentricity orbit with period ranging between 2 and $18 \mathrm{~h}$. The constellation of MEO satellites on circular orbits with inclination $90^{\circ}$ (i.e., going over the North and South Poles) is of interest for our purpose of continuous polar coverage. The MEO orbital period can be also extended beyond $18 \mathrm{~h}$.

The National Oceanic and Atmospheric Administration (NOAA) has considered in the past a MEO constellation as a potential venue for a future satellite system (Dittberner et al. 2004, 2006; Gerber et al. 2005). A technical study conducted by NOAA suggested an eight-satellite circular MEO constellation with altitude around $10400 \mathrm{~km}$ and period equal to $\sim 6 \mathrm{~h}$. The total configuration included a four-satellite constellation in the equatorial plane and four-satellite constellation with inclination $90^{\circ}$ (Gerber et al. 2005). It was concluded that this configuration could provide global continuous coverage with the VZA limit of $85^{\circ}$.

From current prospective, the VZA limit of $85^{\circ}$ (and even $70^{\circ}$ ) is considered too large for many important applications including some key products, such as the atmospheric motion vector (AMV) maps derived from tracking displacements of radiance features in the imagery. Presently, there is a consensus that the maximum VZA for AMV retrievals is in the range $62^{\circ}-$ $68^{\circ}$ depending on error tolerance and implementation approach (NOAA 2016; Hernandez-Carrascal and Bormann 2014). As such, the MEO configuration previously discussed by the NOAA needs reassessment in terms of VZA limit. For the same reason, the HEO constellation requires additional analysis. The NOAA requirement for image acquisition in the tropics and midlatitude zone from a MEO system may be relaxed, as this region is well observed by the meteorological GEO satellite network.

After initial analyses that showed the potential feasibility to achieve continuous global coverage through the combination of GEO and HEO (or MEO) observations, it became clear that the orbital configurations proposed earlier may require some adjustments in terms of VZA limit and overlap with the GEO constellation coverage zone as shown in Fig. 1. The VZA value $55^{\circ}$ is the smallest upper limit for some retrievals, such as snow cover and green vegetation fraction (NOAA 2016). The VZA value $62^{\circ}$ is a limit for several key cloud property and AMV retrievals. The VZA value $70^{\circ}$ is a limit for the retrieval of most other remaining parameters from GEO and LEO satellites, as well as for the direct assimilation of radiances.

Typical VZA limits and corresponding maximum latitude values employed by main GEO satellite operators are summarized in Table 1. From quick analysis of these values, it seems logical to expect an optimal demarcation line to be within the latitude zone of $45^{\circ}-50^{\circ}$ (i.e., around the midpoint between equator and pole). 


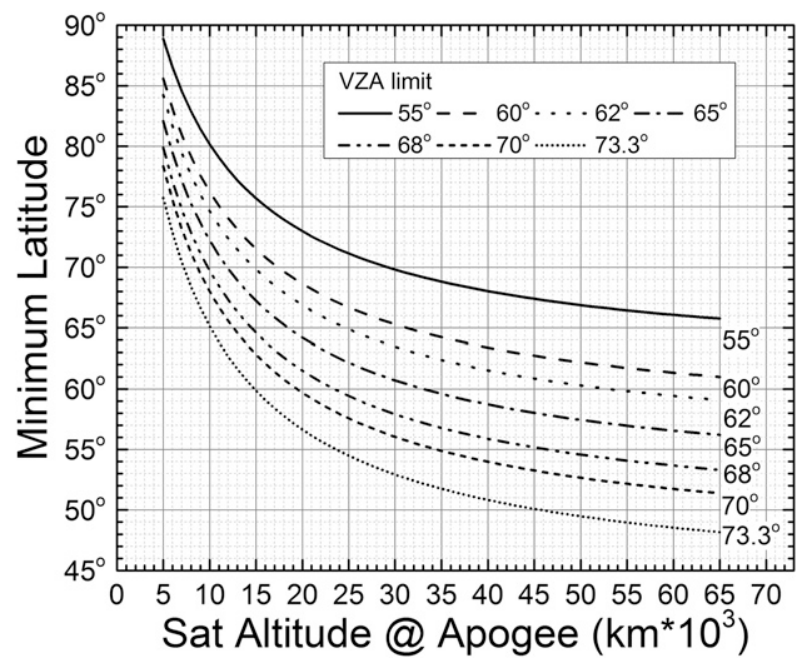

FIG. 3. Minimum latitude viewed across the pole from the apogee point of a HEO satellite at critical inclination $i=63.435^{\circ}$.

This position ensures an acceptable value of VZA for retrievals based on GEO observations. The question now is how to meet this requirement for VZA and coverage zone from a HEO or MEO system?

This study provides a new in-depth analysis of spatial coverage from the HEO and MEO systems as function of the VZA limit and the desired latitude boundary. We also analyzed the ionizing space radiation environment. The key focus is on achieving a satisfactory overlap between GEO and HEO (or MEO) satellite constellations for the VZA values considered as practical limit by operational agencies for various applications.

\section{HEO constellation}

Previous studies of the polar region coverage from HEO have considered a VZA limit equal to $85^{\circ}$ for communications (Chobotov 2002) and $70^{\circ}$ for meteorological imaging (Trishchenko et al. 2016). The classical 12-h Molniya and 14-, 15-, and 16-h highly elliptical orbits evaluated by Trishchenko et al. (2019) are considered in this section as a reference scenario. Based on our analysis, these orbits include the most practical orbital solutions in terms of image acquisition. A baseline HEO configuration should include at least two satellites to achieve continuous coverage of the polar zone. Two satellites configured $180^{\circ}$ apart in the mean anomaly angle (MAA) $\nu$ (Fig. 2) will interchange at the apogee point so that the polar zone can always be observed. It is clear that the minimum (the southernmost) latitude $\Phi_{\text {ap }}$ observed at the apogee point in the Northern Hemisphere by the HEO satellite looking across the North Pole can never be lower than
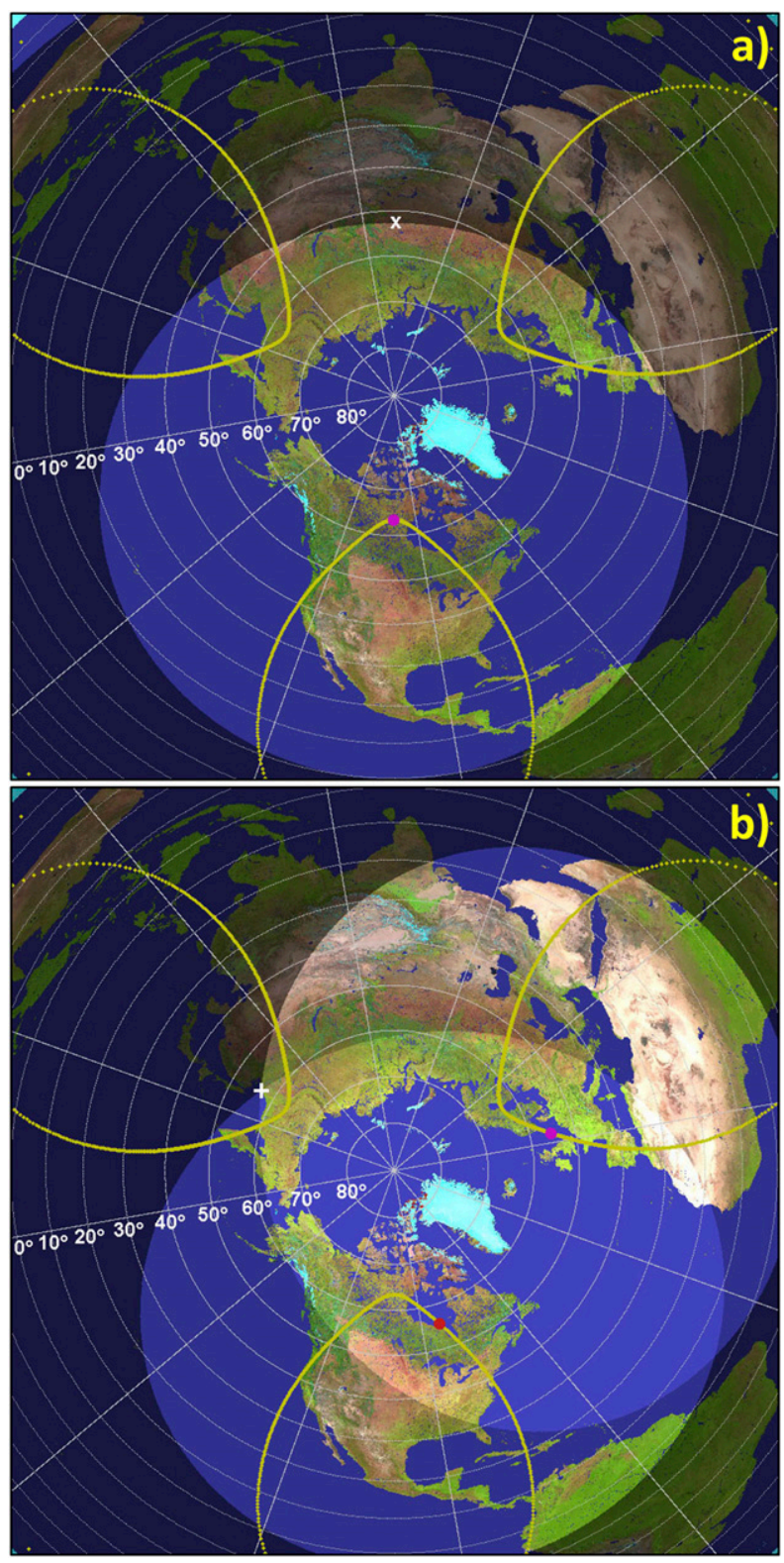

FIG. 4. Example of FOV for the 16-h TAP HEO, $e=0.74$, VZA limit of $70^{\circ}$ : (a) FOV at the apogee point, with the white $\mathrm{x}$ denoting the minimum observed latitude, and (b) overlapping FOVs for a two-satellite constellation when ascending and descending satellites are in the symmetric position $\pm 90^{\circ}$ in the MAA, with the white $\mathrm{x}$ denoting the latitude limit provided by the satellite constellation. The position of the satellite(s) is indicated by a red dot.

$$
\Phi_{\mathrm{ap}}=180^{\circ}-63.435^{\circ}-\varphi_{\mathrm{ap}}=116.565^{\circ}-\varphi_{\mathrm{ap}},
$$

where $\varphi_{\text {ap }}$ denotes the latitude span of the FOV at the apogee point (Fig. A1). It is assumed that the satellite orbit is at critical inclination $i=63.435^{\circ}$. Figure 3 shows values of $\Phi_{\mathrm{ap}}$ as a function of the satellite altitude at the apogee point for several values of VZA. Figure 4a shows 
an example of the FOV map corresponding to VZA limit of $70^{\circ}$ at the apogee point for the 16-h TAP orbit with eccentricity $e=0.74$ described in Trishchenko et al. (2019). The satellite ground track is displayed as yellow contour. The satellite altitude at the apogee is $49600 \mathrm{~km}$ and the starting altitude of imaging is $30450 \mathrm{~km}$ (Table 2). The position of the satellite is indicated by a red dot. The coverage area within VZA limit of $70^{\circ}$ is highlighted in brighter colors over the darker background. The white $\mathrm{x}$ marks the position of a minimum latitude $\Phi_{\mathrm{ap}}$. It is very clear that the area of continuous coverage from the two-satellite HEO system cannot be lower than the $\Phi_{\text {ap }}$ latitude. In fact, it should be slightly higher by a few $\left(\sim 4^{\circ}-5^{\circ}\right)$ degrees, because it corresponds to the intersection of the FOV from the ascending and descending satellites in the symmetric position $\pm 90^{\circ}$ in $\nu$, as shown in Fig. $4 \mathrm{~b}$.

Figure 3 (with correction of about $5^{\circ}$ in latitude) and Fig. 4 prove that a two-satellite HEO constellation cannot deliver continuous coverage below $\sim 55^{\circ}$ latitude with $\mathrm{VZA}<70^{\circ}$ for any practical orbital configuration with the apogee altitude of less than $65000 \mathrm{~km}$. For the VZA limit of $65^{\circ}\left(55^{\circ}\right)$ this can never be lower than $\sim 60^{\circ}\left(70^{\circ}\right)$ latitude. Therefore, a realistic two-satellite HEO constellation cannot solve the problem of continuous coverage for the latitude band $45^{\circ}-50^{\circ}$ (i.e., requirement identified in section 1), even for the VZA limit of $70^{\circ}$. Will this requirement be satisfied if a third satellite is added to HEO constellation?

The spatial coverage from a three-satellite HEO constellation benefits most when the satellites are placed $240^{\circ}$ apart in the MAA denoted as $\nu$ in Fig. 2, and the right ascension of ascending node (RAAN), denoted as $\Omega$, differs by $120^{\circ}$; that is, satellites are placed in three orbital planes (Chobotov 2002). We call this configuration P3. The configuration with all three satellites separated in $\nu$ by $120^{\circ}$ and placed in one orbital plane (configuration P1) does not improve significantly the spatial coverage relative to the twosatellite configuration. In addition, configuration P3 increases the number of apogee positions, thus leading to more uniform distribution of viewing conditions (except for the 12-h Molniya HEO constellation). This is shown in Figs. 5a-c. Figures $5 \mathrm{a}$ and $5 \mathrm{~b}$ display a combined FOV for the three-satellite configuration P3 for the 16-h TAP orbit $(e=0.74)$ already discussed above. Figure 5a displays the combined FOV for constellation P3 when the latitude of the area outside of combined FOV reaches a maximum, that is, corresponds to the lowest latitude of continuous coverage. Figure $5 \mathrm{~b}$ displays the combined FOV for constellation $\mathrm{P} 3$ when two satellites are positioned $\pm 60^{\circ}$ relative to

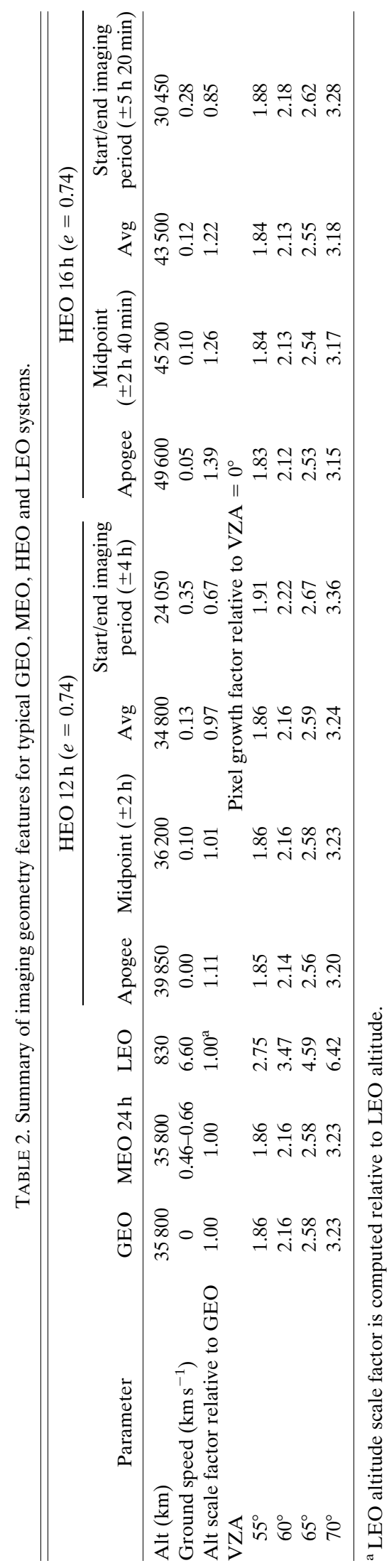



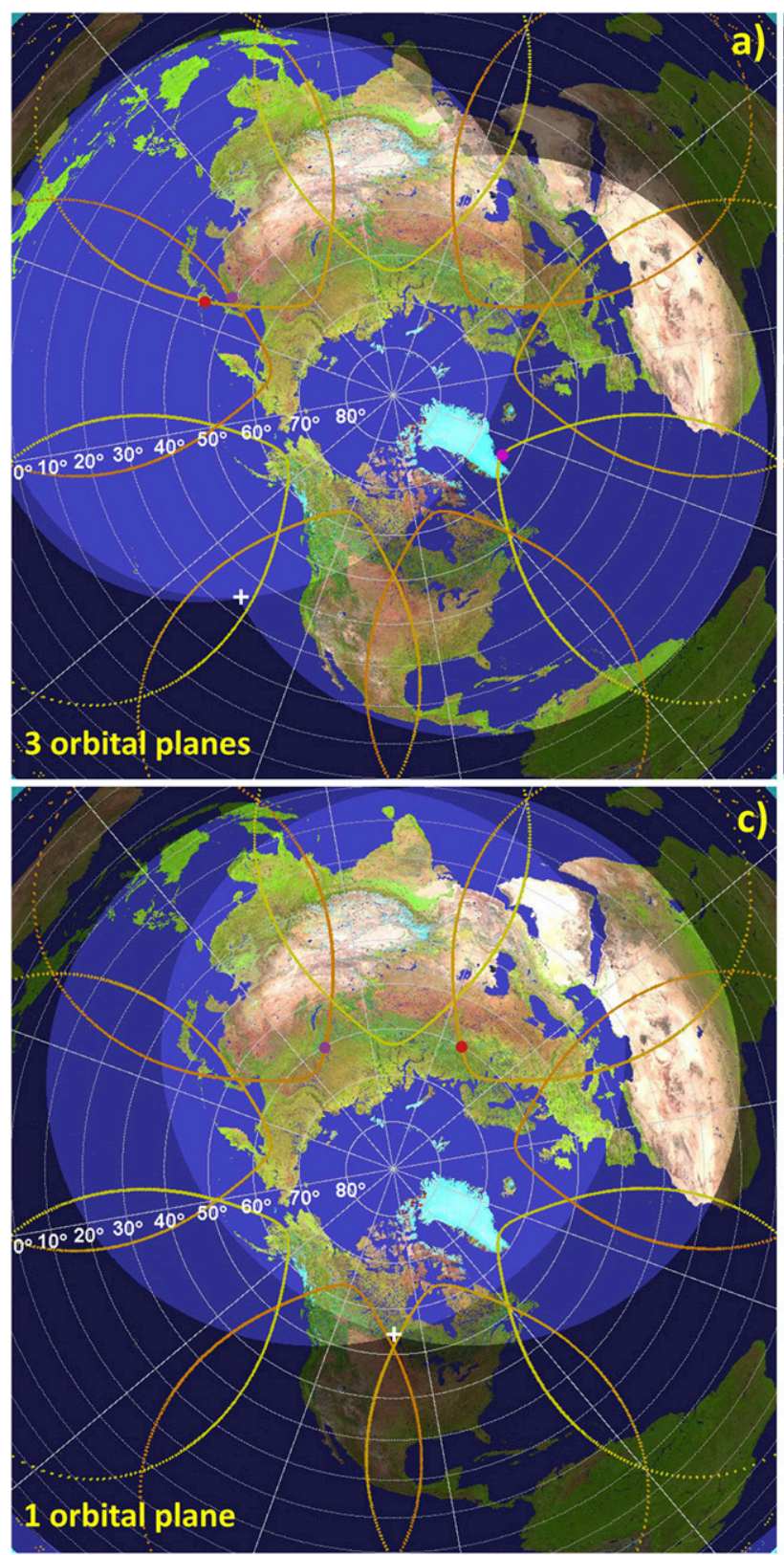

direction to apogee $\left(\nu=180^{\circ}\right)$ and the third satellite is at the perigee point $\left(\nu=0^{\circ}\right)$. It is interesting to note that this situation (i.e., when effectively only two satellites observe the polar region of interest) provides slightly better coverage in terms of maximum latitude of the area outside of combined FOV relative to configuration shown in Fig. 5a when all three satellites observe the region. Figure $5 \mathrm{c}$, which corresponds to $\mathrm{HEO}$ configuration $\mathrm{P} 1$ with satellites positioned, as in Fig. $5 \mathrm{~b}$ in terms of $\nu$, displays a configuration where the lowest latitude of continuous coverage is achieved. The comparison of results from

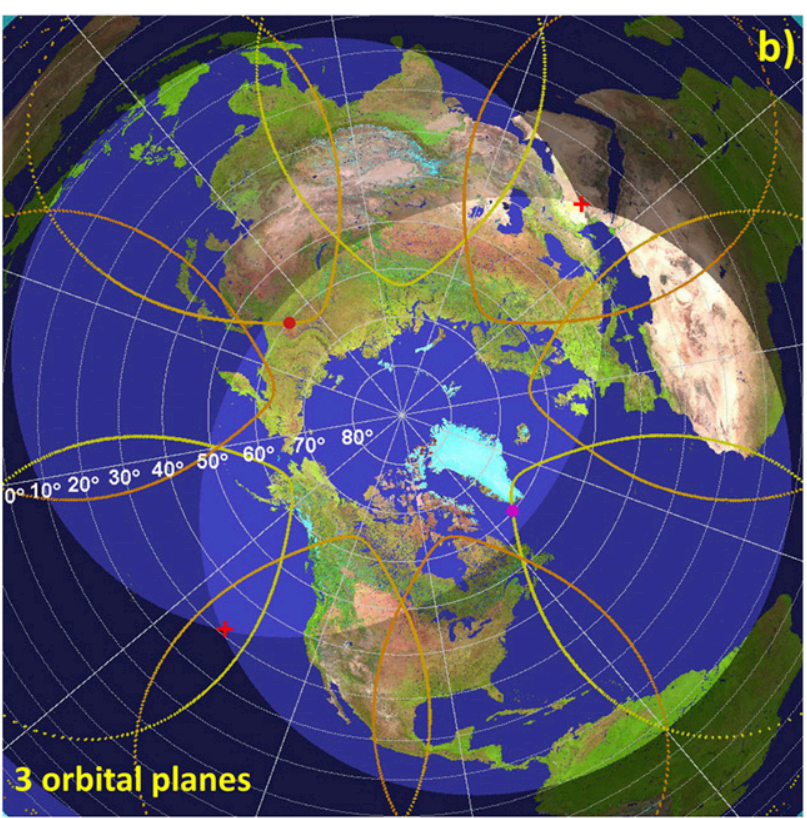

FIG. 5. Comparison of spatial coverage from three-satellite 16-h TAP HEO constellation in three orbital planes $\left(120^{\circ}\right.$ apart in RAAN; P3) as shown in (a) and (b) vs constellation in one orbital plane P1 as shown in (c): (a) Earth views for a minimum latitude of continuous observations, (b) Earth views from satellites $\pm 60^{\circ}$ in MAA around the apogee direction (two-satellite viewing), and (c) as in (b), but for HEO constellation in one orbital plane. White or red plus signs denote the highest value of latitude outside of combined FOV. Here, the VZA $=70^{\circ}$.

Figs. $4 \mathrm{~b}$ and $5 \mathrm{c}$ show that configuration $\mathrm{P} 1$ with all three satellites in one orbital plane and separated by $120^{\circ}$ in $\nu$ provides only a marginal improvement over the two-satellite HEO configuration.

Figure 6 summarizes spatial coverage results for one-, two-, and three-satellite HEO constellations by displaying zonal mean percentage of daily coverage as a function of latitude for different VZA values: $70^{\circ}, 62^{\circ}$, and $55^{\circ}$. Results are displayed for the Northern Hemisphere only. Statistics have been computed on $1^{\circ} \times 1^{\circ}$ spatial global map from satellite orbits modeled at 1 -min temporal resolution over duration of several orbital 


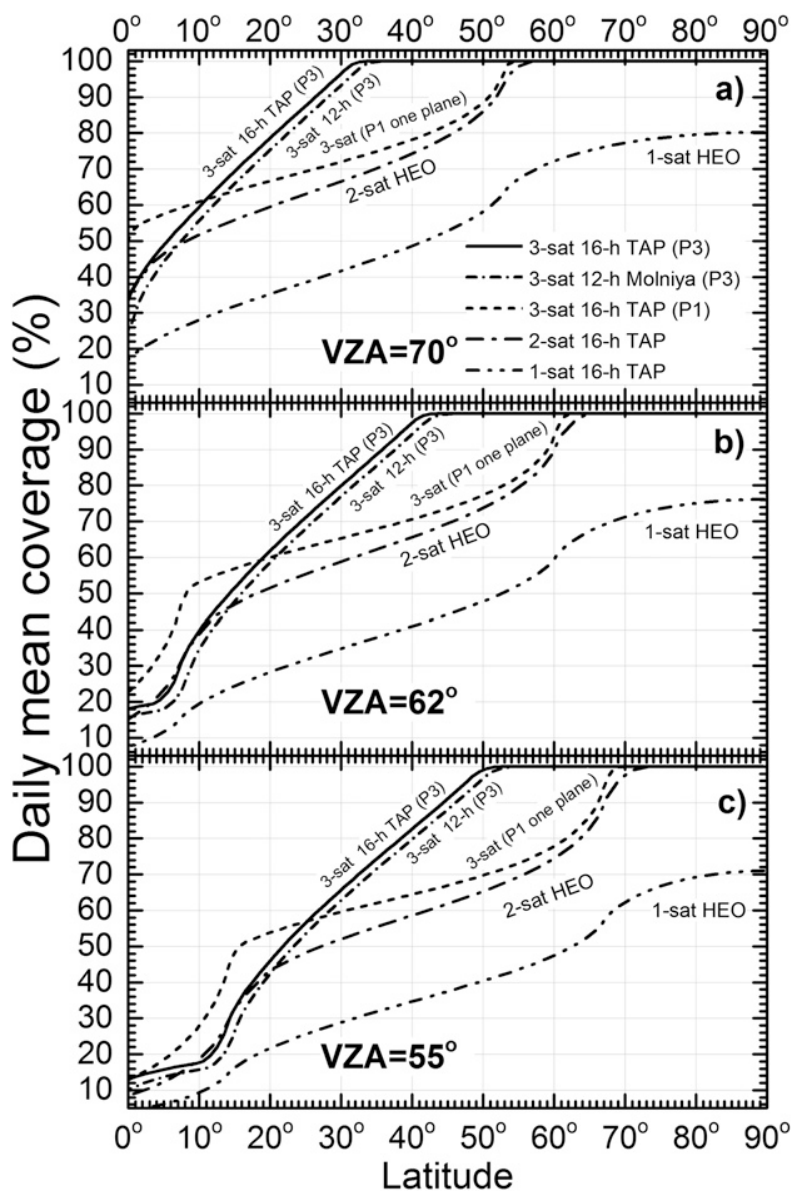

FIG. 6. Daily zonal mean coverage from one-, two-, and three-satellite HEO systems for various VZA limits: (a) $70^{\circ}$, (b) $62^{\circ}$, and (c) $55^{\circ}$.

cycles. Results for the 16-h TAP and 12-h Molniya orbits are shown. Zonal mean statistics are fairly close over the region of main interest (latitude $>40^{\circ}$ ), which is explained by the similarity of the orbital shape and the imaging altitude ranges. The coverage from 16-h TAP orbit is slightly better because of slightly higher imaging altitude on average (Table 2). Results for other orbits, such as 14- and 15-h orbits described in Trishchenko et al. (2019), fall between curves for 12- and 16-h orbits. The main conclusion that can be drawn from Fig. 6 is that the zone of continuous imaging from the threesatellite system for VZA limit $70^{\circ}$ can be extended down to $33^{\circ}-35^{\circ} \mathrm{N}$ in comparison with $58^{\circ}-60^{\circ} \mathrm{N}$ for a twosatellite $\mathrm{HEO}$ system. Corresponding improvements are $42^{\circ}-44^{\circ} \mathrm{N}\left(52^{\circ}-54^{\circ} \mathrm{N}\right)$ versus $64^{\circ}-66^{\circ} \mathrm{N}\left(72^{\circ}-74^{\circ} \mathrm{N}\right)$ for a VZA limit of $62^{\circ}\left(55^{\circ}\right)$. One can also see that configuration $\mathrm{P} 1$ when all satellites are placed in one orbital plane and separated by $120^{\circ}$ in $\nu$ provides only a marginal improvement over the two-satellite system. This can be explained by a significant degree of FOV overlap between satellites in P1 configuration, as already mentioned in relation with Fig. 5. Configuration P3 creates a polar zone viewing from two of the three satellites located $180^{\circ}$ apart in longitude that maximizes simultaneous imaging. Spatial coverage from a one-satellite HEO system is also shown in Fig. 6 for reference.

Configuration P3 with three orbital planes leads to the multiple-apogee locations for all considered orbits (14-, 15-, and 16-h TAP), except for the 12-h Molniya HEO. In this latter case, satellites are phased $8 \mathrm{~h}\left(240^{\circ}\right.$ in $\left.\nu\right)$ apart and separated in $\Omega$ by $120^{\circ}$, which leads to repeating the ground track. Multiple-apogee locations $(12 \times 3=36$ for 14-h HEO over 7 days, $8 \times 3=24$ for 15 -h HEO over 5 days, and $3 \times 3=9$ for 16 -h HEO over 2 days) lead to relatively uniform longitudinal geographical distribution of the spatial coverage. It depends mostly on the latitude (zonal symmetry). In the case of 12-h Molniya orbit, the map of spatial distribution appears very uneven and centered around the ground track. Figure 7 further

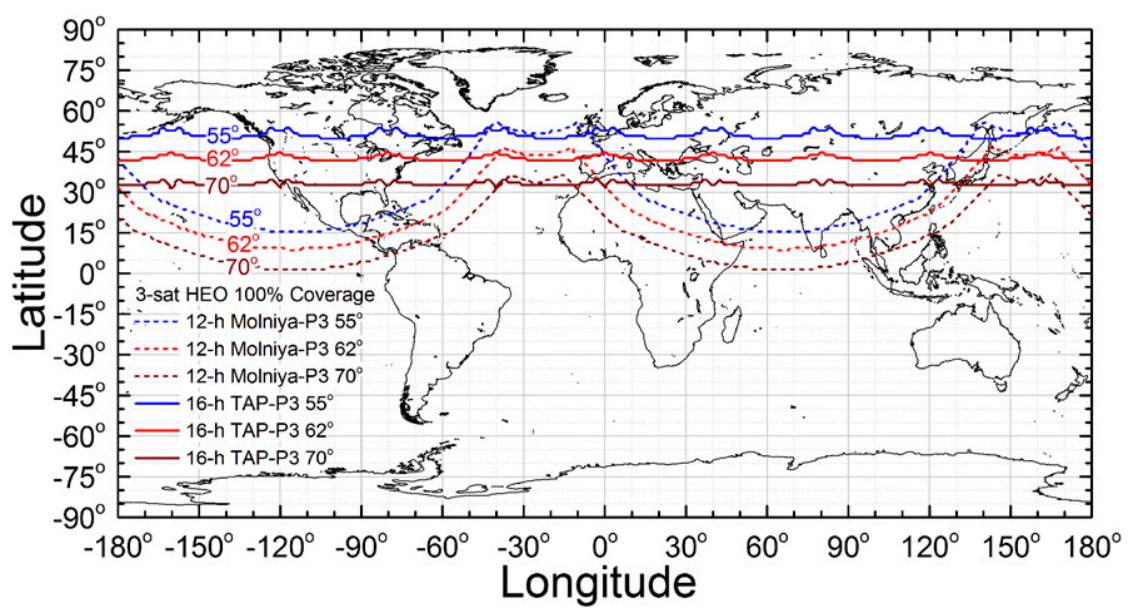

FIG. 7. Delineation of continuous (100\%) spatial coverage for 16- and 12-h three-satellite HEO constellations and VZA limits $55^{\circ}, 62^{\circ}$, and $70^{\circ}$. 

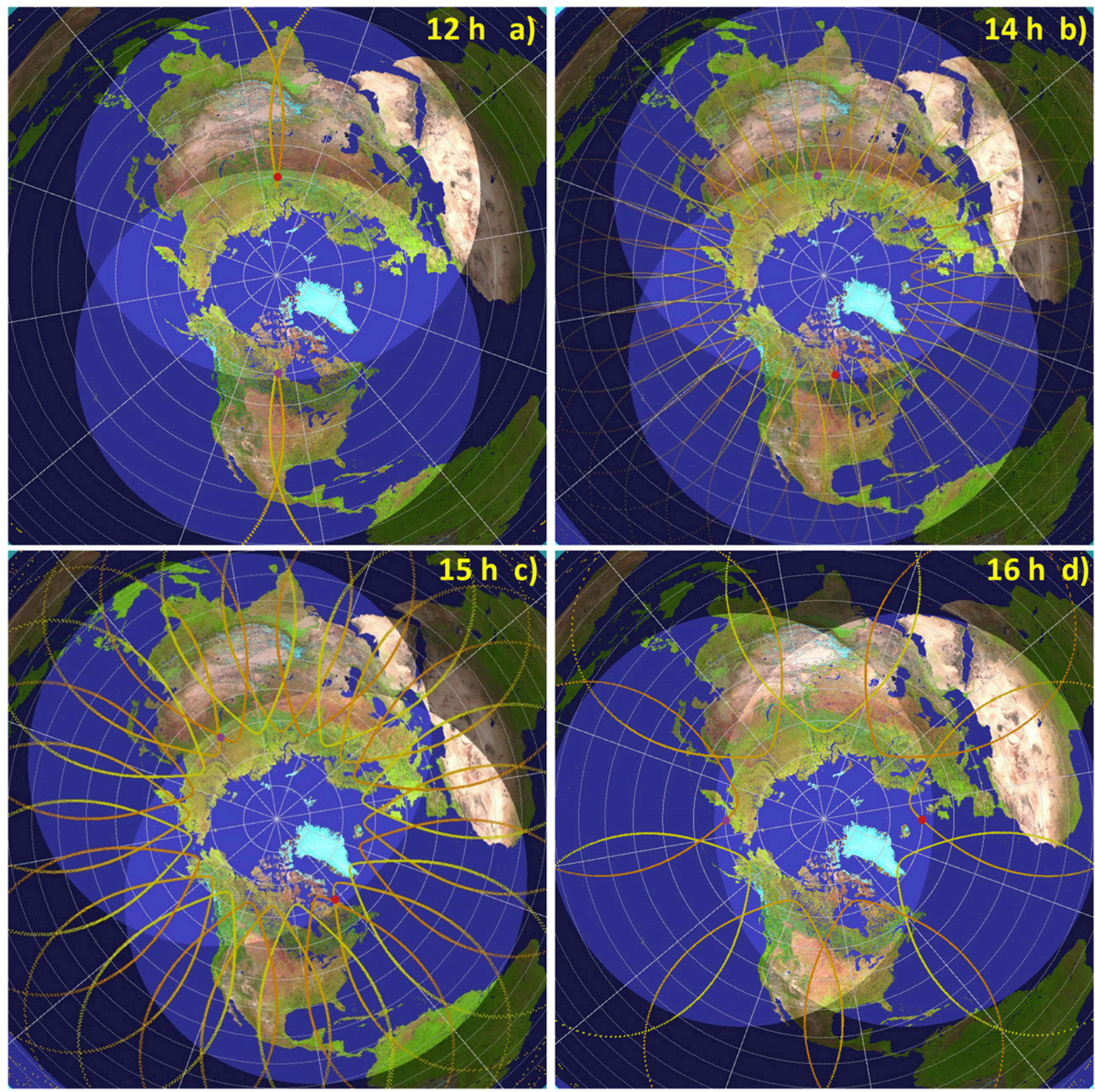

FIG. 8. Apogee distribution for three-satellite HEO constellations in three orbital planes P3: (a) 12 (Molniya orbit), (b) 14, (c) 15, and (d) $16 \mathrm{~h}$ (TAP). The configuration of satellite positions that is shown corresponds to MAA values $\nu=0^{\circ}$ (perigee), $120^{\circ}$, and $240^{\circ}$. Molniya HEO satellites follow the same ground track with two apogee positions despite three orbital planes.

illustrates these features. It shows $100 \%$ isolines for spatial coverage from three-satellite 12-h Molniya and 16-h TAP HEO constellations P3 for the VZA values $55^{\circ}, 62^{\circ}$, and $70^{\circ}$. Zonal mean distributions are fairly close (Fig. 6), but spatial maps, as shown in Fig. 7, look very different. The spatial distribution for multiapogee 16-h TAP HEO system is close to zonal, whereas the 12-h Molniya HEO system allows continuous imaging for extensive areas located in the tropical zone. The coverage for $70^{\circ} \mathrm{VZA}$ may extend almost to equator for the 12-h Molniya HEO system. It seems possible to configure P3 12-h Molniya HEO constellation so that it can observe the entire North American and Eurasia landmasses within VZA limit of $55^{\circ}$. This enhanced spatial coverage is achieved at the expense of significant unobserved areas located between two branches of the ground track (i.e., perpendicular to the center line in Fig. 8a) and restricted range of viewing conditions when observations are acquired from the satellite positioned within a relatively small region (i.e., quasi-GEO viewing 
conditions near apogee). The multiple-apogee systems, as shown in Figs. 8b-d, demonstrate a significant degree of zonal symmetry and a wide range of viewing conditions over the entire ground track repeat cycle.

\section{MEO constellation}

Equations (A8) and (A13) from appendix A define the number of satellites in the MEO constellation that can achieve continuous coverage poleward of a given latitude (low boundary) and within specified VZA limit. Assumptions about the MEO satellite constellation used for the derivation of equations in appendix A assumed spherical Earth, orbit inclination $90^{\circ}$, and a MEO configuration in one orbital plane with spacecraft equally phased in $\nu$. This ensures that overlapping between two FOVs from adjacent imagers always embraces the required latitude range (defined by parameter $\varepsilon$ in Fig. A2 of appendix A). Corresponding results for the number of satellites in the MEO constellation are presented in Fig. 9. Three panels display results for three VZA limits of $55^{\circ}, 62^{\circ}$, and $70^{\circ}$. The number of satellites is shown as a function of satellite altitude (period) for various values of low boundary latitude. The range of altitudes in Fig. 9 extends up to $55000 \mathrm{~km}$ (equivalent period of about $42 \mathrm{~h}$ ). Results for the polar area with low boundary located within latitude range between $45^{\circ}$ and $50^{\circ}$ are highlighted in pink color. One can see that in some cases the area shrinks into one line because of the nature of results shown in Fig. 9, which are rounded to integers.

Figure 9 demonstrates that not less than four MEO satellites are required to achieve continuous coverage of the polar region from altitudes below $54000 \mathrm{~km}$ (or equivalent period of about $41 \mathrm{~h}$ ) for the VZA limit $70^{\circ}$ (Fig. 9c). The latitudes above $60^{\circ}\left(45^{\circ}\right)$ can be observed within VZA of $70^{\circ}$ from the altitudes $\geq 14000 \mathrm{~km}$ $(\geq 29000 \mathrm{~km})$ with corresponding periods $\geq 8 \mathrm{~h}(\geq 18 \mathrm{~h})$. Figure 9b shows that four MEO satellites in constellation can also achieve continuous coverage within VZA limit of $62^{\circ}$ but the latitudinal boundary cannot be lower than $\sim 55^{\circ}$ and satellite altitudes should be $\geq 38000 \mathrm{~km}$ (period $\geq 25.8 \mathrm{~h}$ ). Four MEO satellites with periods $\leq 24 \mathrm{~h}$ (altitudes $\leq 36000 \mathrm{~km}$ ) can achieve continuous coverage at the VZA limit $62^{\circ}$ only above $\sim 60^{\circ}$ latitude.

Five MEO satellites are required to achieve continuous coverage at the VZA limit of $55^{\circ}$ according to Fig. 9a. To cover the boundary at latitude of $60^{\circ}$ at the VZA limit of $55^{\circ}$ the satellites should fly higher than $26000 \mathrm{~km}$ (period $\geq 16 \mathrm{~h}$ ). For the satellite altitudes $\geq 40000 \mathrm{~km}$ (period $\geq 27.6 \mathrm{~h}$ ) continuous coverage within VZA limit $55^{\circ}$ with five satellites can be achieved down to $55^{\circ}$ latitude. On the other hand, no fewer than six satellites are

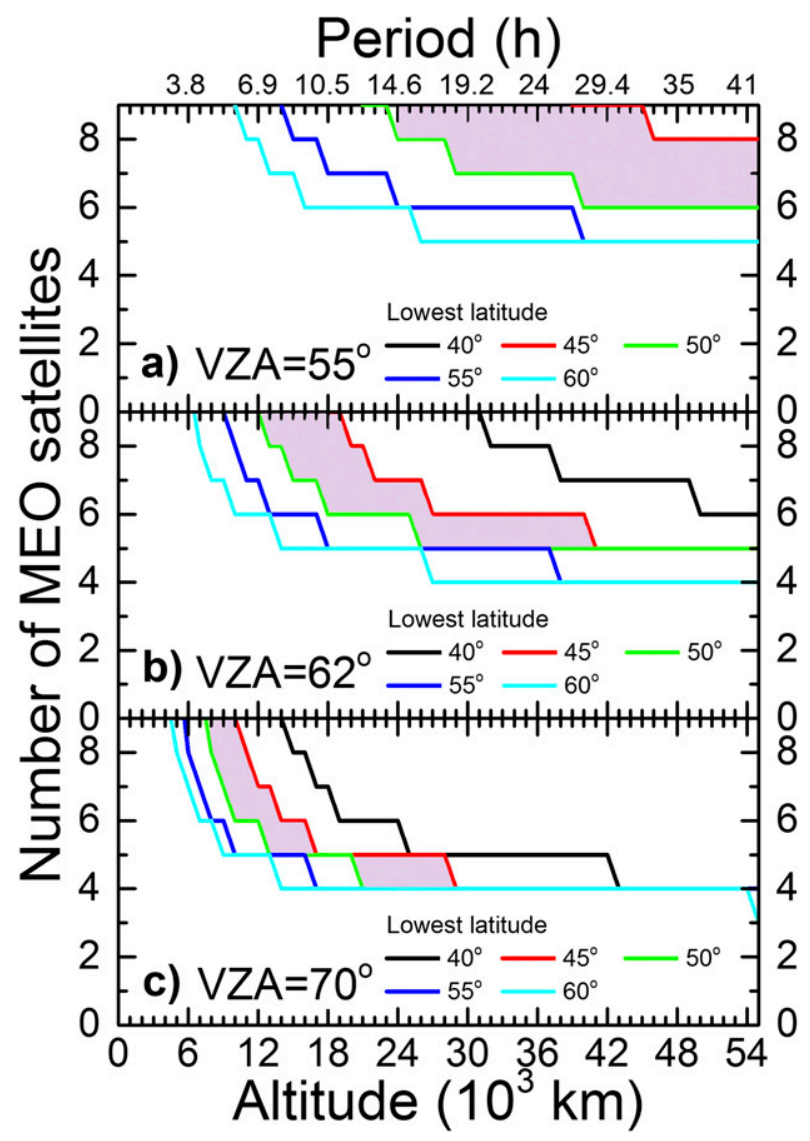

FIG. 9. Number of satellites in the MEO constellation for various VZA and low boundary latitude as function of orbit altitude: VZA = (a) $55^{\circ}$, (b) $62^{\circ}$, and (c) $70^{\circ}$. The pink-colored region highlights results for low boundary position within the latitude zone $45^{\circ}-50^{\circ}$.

required to achieve continuous coverage at the VZA limit of $55^{\circ}$ for the latitude zone above $55^{\circ}$ with lowest altitudes starting at $24000 \mathrm{~km}$ (period $\geq 14.6 \mathrm{~h}$ ).

To achieve the MEO spatial coverage approximately similar to the three-satellite HEO constellation in terms of low boundary position and VZA limit, the MEO constellation with period $\leq 24$ h (i.e., GEO-type or better imaging conditions) should include six satellites in the constellation. As a brief general recommendation based on results presented in Fig. 9, a valuable option is a six-satellite MEO constellation with orbital period of $24 \mathrm{~h}$. This system provides viewing conditions that are similar to GEO satellites equipped with newgeneration GEO imagers, such as Advanced Baseline Imager (ABI) and its modifications, developed and operated by NOAA (Schmit et al. 2005, 2017), Japan Meteorological Agency (JMA), and Korea Meteorological Administration (KMA), as well as the future Flexible Combined Imager (FCI) being developed for the European Meteosat Third Generation (MTG) geostationary spacecraft. 


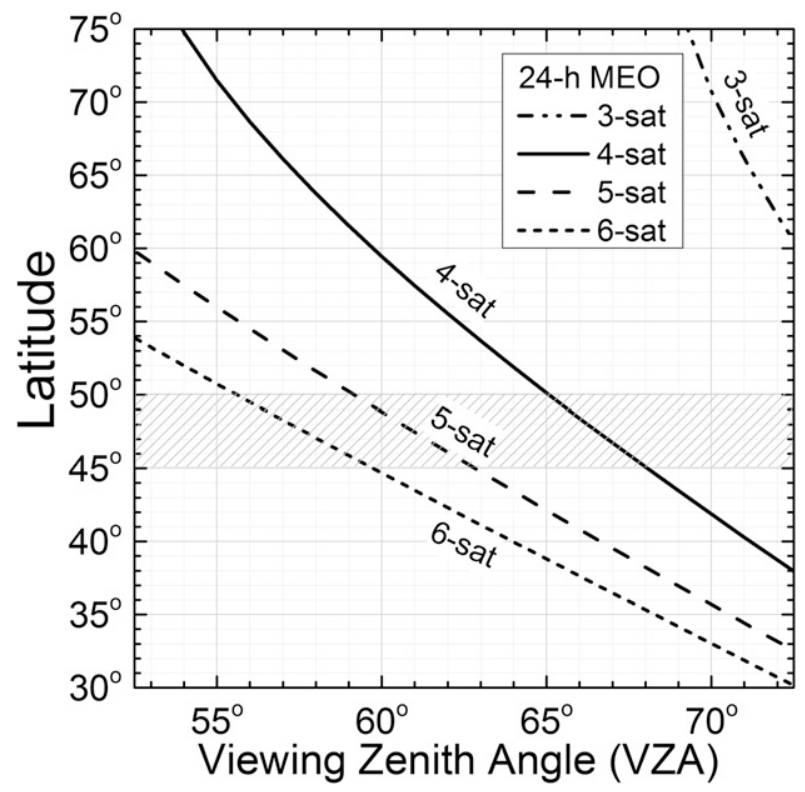

FIG. 10. The low boundary latitude of continuous coverage zone for 24-h MEO constellations with three, four, five, and six satellites as a function of the VZA limit. The latitude zone $45^{\circ}-50^{\circ}$ is highlighted.

Figures 10 and 11 show some additional details about spatial coverage from the MEO constellations with three, four, five, and six satellites. Figure 10 displays the low boundary latitude of continuous coverage zone for 24-h MEO constellations as a function of maximum VZA. It clearly shows that a spatial coverage performance similar to that from a three-satellite HEO system can be achieved by a six-satellite MEO constellation with a $24 \mathrm{~h}$ orbital period. Five MEO satellites can also provide very good performance, but the latitude range at $\mathrm{VZA} \leq 60^{\circ}$ is slightly worse than that achieved from HEO. Although the MEO constellation contains twice the number of satellites relative to the HEO system, it provides continuous imaging for both poles. In this regard, both systems are equivalent. A slight disadvantage of the MEO system is its relatively large ground speed $\left(\sim 0.5 \mathrm{~km} \mathrm{~s}^{-1}\right)$ as shown in Table 2 . This requires some degree of overlapping between consecutive scans during image acquisition from the MEO platform. On the other hand, MEO imagers acquire Earth views from a constant altitude, which for 24-h circular MEO orbit is lower than altitudes of spacecraft in HEO during a significant part of the imaging period (Trishchenko et al. 2019).

Figure 11 displays the zonal mean daily average coverage from 24-h MEO multisatellite systems. It demonstrates that significant areas of the globe in the midlatitude and tropical zones can be observed for more than $50 \%$ of the time. This capability is beneficial for the MEO-GEO

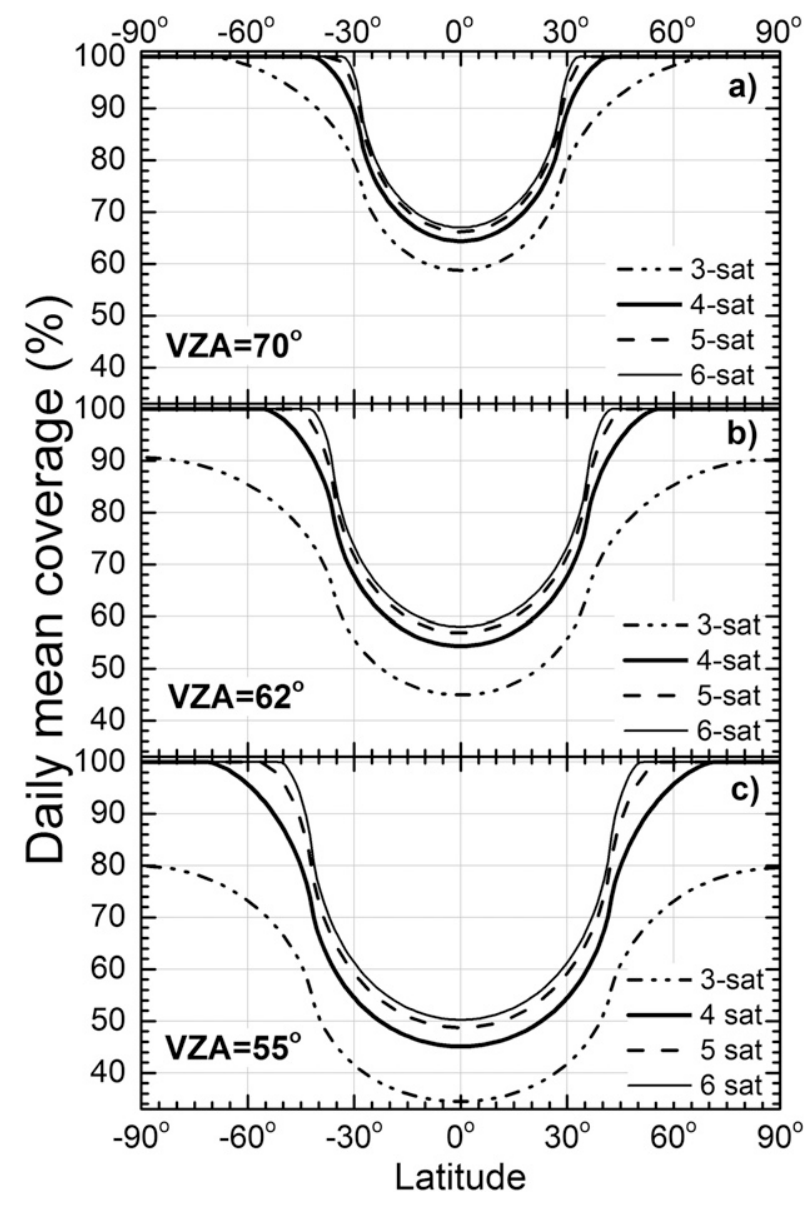

FIG. 11. Daily zonal mean coverage from three-, four-, five-, and six-satellite 24-h MEO systems for various VZA limits: (a) $70^{\circ}$, (b) $62^{\circ}$, and (c) $55^{\circ}$.

and MEO-LEO satellite intercalibration, as well as for potential use in improved retrievals of scene anisotropy (bidirectional reflectance/emission function), cloud height and AMV among others, from multiview (stereo) observations. Figure 11 also confirms that a sixsatellite MEO constellation provides a relatively small enhancement in spatial coverage over the five-satellite constellation in comparison to four-to-five MEO satellite enhancement. However, this small enhancement is important to achieve performance similar to HEO in the latitude area of $45^{\circ}-50^{\circ}$. Some additional trade-off studies may be required to evaluate the exact number of MEO satellites (five or six) in the constellation to meet various observational needs.

\section{Space radiation environment}

Space radiation environment is among key factors influencing the space mission design. We modeled it for all considered types of orbit with periods of $\leq 24 \mathrm{~h}$ using 
the European Space Agency (ESA) Space Environment Information System (SPENVIS) (Heynderickx et al. 2004). The SPENVIS model setup follows that one presented in Trichtchenko et al. (2014) and Trishchenko et al. (2019) and is briefly summarized below. The radiation models for the trapped protons AP8 and electrons AE8 (Sawyer and Vette 1976; Vette 1991) have been utilized for conditions of a solar maximum for consistency with our previous analysis providing conservative estimates. The solar proton fluences have been computed using SPENVIS built-in "ESP-PSYCHIC" model with setup for the worst event (Xapsos et al. 1999, 2000). The total ionizing dose and its components have been estimated using SPENVIS built-in model "SHIELDOSE" (Seltzer 1980) with shielding configuration for the finite aluminum slab and the silicon $(\mathrm{Si})$ as target material. The logarithmic values of ionizing dose have been interpolated to derive shielding thickness for an annual total ionizing dose (TID) value selected equal to $3.33 \mathrm{krad}$, which corresponds to accumulated TID equal to $50 \mathrm{krad}$ for 15-yr space mission (Emmanuel et al. 2014; Trichtchenko et al. 2014; Trishchenko et al. 2019).

Figure 12 displays results of SPENVIS modeling. The aluminum thickness to achieve $50 \mathrm{krad}$ TID for a mission lifetime of 15 years is plotted as a function of orbital period for MEO missions ( 6 to $24 \mathrm{~h}$ ) as well as for the classical 12-h Molniya and for 14-, 15-, and 16-h HEO systems considered earlier. The top $x$ axis also shows tick labels for the equivalent circular MEO altitude. This altitude scale cannot be directly applied to the HEO system because of variable orbital altitude. Some specific details of orbital altitude variations for selected HEO systems can be found in Trishchenko et al. (2011, 2016, 2019), Trishchenko and Garand (2011), and in Table 2. The curve for MEO systems has a local minimum of ionizing radiation at $\sim 8000 \mathrm{~km}$ (equivalent period of about $5 \mathrm{~h}$ ) that corresponds to location of a slot region between the inner and the outer electron belts. The MEO systems at lower altitudes (i.e., shorter periods) pass the inner electron radiation belt and also penetrate deeply into the trapped proton region. This is why they show steep increase in aluminum shielding thickness from 3.9 to $4.8 \mathrm{~mm}$ when the MEO satellite altitude reduces from 8000 to $6000 \mathrm{~km}$. The overall maximum around $5 \mathrm{~mm}$ of shielding in Fig. 12 is achieved for the MEO systems at $16000 \mathrm{~km}$ altitude (period $\sim 9 \mathrm{~h}$ ). It corresponds to the central region of the outer electron belt. After that point, the shielding thickness monotonically decreases. At the end of the curve at the altitudes around $36000 \mathrm{~km}$ (period $24 \mathrm{~h}$ ), it reduces to $2.2 \mathrm{~mm}$ (i.e., $44 \%$ of the maximum). The selection of 24-h MEO system ensures the most favorable space radiation environment among other MEO systems with periods of $\leq 24 \mathrm{~h}$.

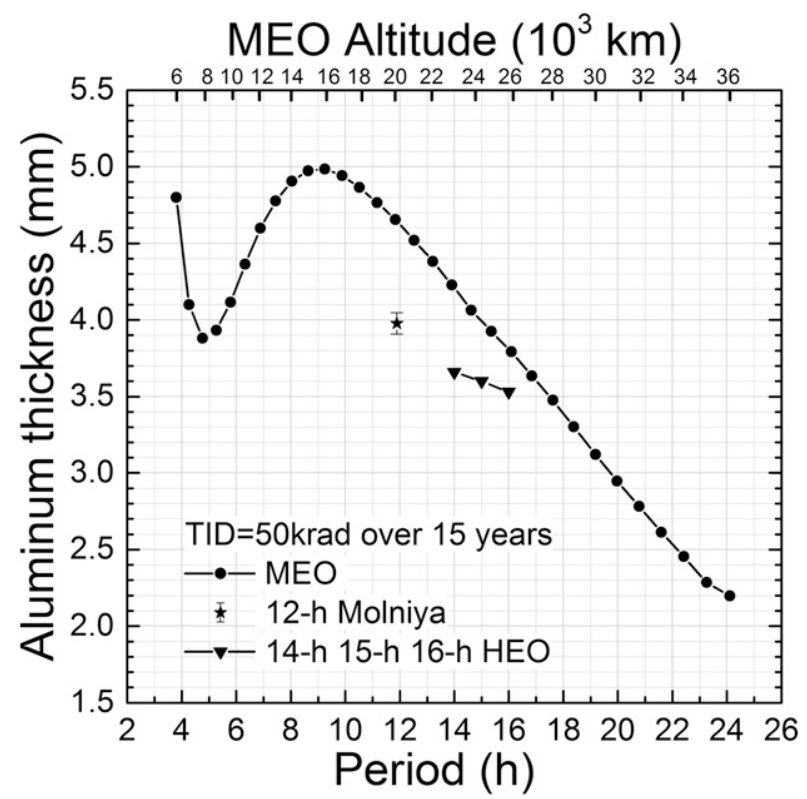

FIG. 12. The thickness of aluminum slab to keep the annual TID at $3.33 \mathrm{krad} \mathrm{yr}^{-1}$, i.e., 15 years of mission lifetime for a total mission dose of $50 \mathrm{krad}$. Results are shown for the range of MEO orbits with altitudes between 6000 and $36000 \mathrm{~km}$ (periods from 3.8 to 24.1 h), 12-h Molniya HEO, and the set of 14-, 15-, and 16-h orbits described by Trishchenko et al. (2019). The SPENVIS tool with AP-8/AE-8 models was used.

The 12-h Molniya orbit requires about $4 \mathrm{~mm}$ of aluminum shielding for selected TID level. Some variations in the shielding thickness shown by margin bars are due to the selection of different RAAN values (i.e., relative orientation of the orbital plane with respect to Earth's magnetic field). The 12-h Molniya HEO system has only two apogees. To account for the possible spread of radiation environment conditions depending on specific selection of the RAAN parameter, we conducted simulations for all possible RAAN angles $0^{\circ}-360^{\circ}$ with $45^{\circ}$ steps and then computed average $(\sim 3.98 \mathrm{~mm})$, maximum $(4.02 \mathrm{~mm})$, and minimum $(3.91 \mathrm{~mm})$ values, which are displayed in Fig. 12. Figure 12 also shows results for 14-, 15-, and 16-h HEO systems. They are 3.66, 3.60, and $3.53 \mathrm{~mm}$, correspondingly. Figure 12 demonstrates that from the radiation point of view, the 24-h MEO constellation has an advantage over HEO constellations considered in this paper. Among considered HEO systems, the 16-h TAP HEO described in Trishchenko et al. (2019) has a slight advantage from the space radiation environment point of view.

\section{Discussion and conclusions}

The highly elliptical orbit and the medium Earth orbit satellite constellations are compared in this study with 
regard to their capacity for continuous coverage of polar regions within different viewing zenith angle limits and ensuring an adequate image overlap with the GEO satellite network. Until recently, the VZA range in most studies in this field has been set to $70^{\circ}$ for meteorological applications. The latitude of the boundary line has been assumed equal to $60^{\circ}$; that is, continuous coverage above this line should be provided by the polar observing system while the geostationary satellite network is responsible for the area of the globe below this latitude. The detailed analysis of requirements from various international meteorological agencies for retrieval of key meteorological products, such as atmospheric motion vectors, cloud, and surface properties, suggests that the GEO observations should ideally overlap with polar observing system (HEO or MEO) over the latitude band $45^{\circ}-50^{\circ}$ to ensure a reliable transition in retrieved parameters from these two systems. This corresponds to maximum VZA values for the GEO imaging systems ranging between $60^{\circ}$ and $64^{\circ}$, which is adequate for all key retrievals. It is, therefore, important to understand how the HEO or MEO satellite constellations can meet these VZA and latitude coverage requirements.

Building upon previous studies (Trishchenko et al. 2011, 2016, 2019; Trichtchenko et al. 2014; Trishchenko and Garand 2011), we conducted an extended analysis of several realistic HEO and the MEO constellations. We considered the classical 12-h Molniya and 14-, 15-, and 16-h HEO constellations at the critical orbit inclination $i=63.435^{\circ}$ and other orbit parameters described in detail by Trishchenko et al. (2019).

This study has determined that a three-satellite HEO constellation can achieve continuous coverage down to $45^{\circ}-50^{\circ}$ latitude region with a maximum VZA value below $60^{\circ}$. The three-satellite HEO system must be configured to fly in three orbital planes so that their RAAN angles are separated by $120^{\circ}$ and the MAAs are phased $240^{\circ}$ apart. For the 12-h Molniya HEO constellation this leads to a ground track with two apogee points repeated by all three satellites. The ground track of the Molniya HEO system can be positioned to achieve coverage of most of the Northern Hemisphere landmass up to $15^{\circ}$ latitude at the VZA limit $55^{\circ}$ leaving some ocean areas covered at significantly higher latitudes (Fig. 7). The 14-, 15-, and 16-h three-satellite HEO systems provide much more uniform spatial coverage because observations are acquired from multiapogee satellite tracks uniformly distributed around the globe (Fig. 8). This in principle reduces geometry-related biases in the parameter retrievals. The images are acquired from 36 apogee locations for the 14-h HEO system over a 7-day period, 24 apogee locations for the 15-h HEO system over a 5-day period, and 9 apogee locations for the 16-h HEO system over a 2-day period. Two sets of three-satellite HEO constellation are required to provide coverage over both poles.

The analysis of the MEO system with altitudes between 6000 and $55000 \mathrm{~km}$ (equivalent orbital periods between 3.8 and $42 \mathrm{~h}$ ) suggests that HEO-equivalent spatial coverage of polar regions can be optimally achieved from a six-satellite 24-h MEO constellation. The MEO system utilizes circular orbits with $90^{\circ}$ inclination. The images from the 24-h MEO are acquired from a constant altitude similar to GEO in contrast to the HEO case where the orbit altitude varies. Because of the ground speed of the MEO satellites (see Table 2), some adjustment is required in the image scanning to ensure the absence of gaps. This issue is, however, not perceived as a significant technical problem and can be solved for the current third generation GEO-type imagers, such as ABI and FCI. The candidate 24-h MEO constellation provides the coverage of the tropical zone more than $50 \%$ of the time. This is beneficial for satellite intercalibration and for creating the backup imaging capacity in this zone, as well as for improved retrievals due to multiview capacity.

Table 2 summarizes some key imaging geometry features for typical GEO, HEO, MEO, and LEO systems discussed above. It includes the values of altitude, satellite ground speed, altitude scale factor, and pixel growth factor $F_{\mathrm{PGF}}$ as a function of VZA computed according to Eq. (A15) defined in appendix A. The HEO and MEO image viewing is scaled to the GEO altitude. As such, the viewing conditions for 24-h MEO system is identical to GEO, except for the satellite ground speed $\left(0.46-0.66 \mathrm{~km} \mathrm{~s}^{-1}\right)$. It is expected that the suggested MEO system could provide imagery with spatial resolution similar to GEO. The spatial resolution of imagery from the HEO system is also similar to GEO. The altitude scale factor (i.e., the satellite altitude normalized to the GEO altitude) for HEO systems varies between 0.67 and 1.39. The average altitude for the entire imaging period (assuming $16 \mathrm{~h}$ of imaging per day per satellite) and altitude at midpoint of the imaging interval for 12-h Molniya HEO are fairly close to the GEO value. Corresponding altitude scaling factors are 0.97 and 1.01. These values for 16-h TAP HEO system are 1.22 and 1.26. The relative variations of image spatial resolution due to HEO satellite altitude change are significantly smaller than variations of image spatial resolution due to the VZA effect equally applicable to all imaging systems (GEO, MEO and HEO). For example, the pixel growth factor $F_{\mathrm{PGF}}$ for the GEO imaging is equal to 1.86 at $\mathrm{VZA}=55^{\circ}$ and grows to 3.23 for $\mathrm{VZA}=70^{\circ}$. The VZA dependence of $F_{\mathrm{PGF}}$ for the HEO systems is within $2 \%-4 \%$ of the GEO. Table 2 also includes results for the LEO imaging system for reference. It shows that the VZA effect is approximately a factor of 
2 stronger for LEO because of smaller imaging altitude and, therefore, stronger effect of Earth's curvature.

In addition to enhanced imaging capacity, the $\mathrm{HEO}$ an $\mathrm{MEO}$ systems could also provide an enhanced capacity for atmospheric sounding, such as retrieval of temperature and humidity profiles, columnar amounts and profiles of aerosols, greenhouse and other radiatively active gases important for air quality, weather forecasting, and climate applications. This requires high-resolution spectral measurements in the thermal infrared (IR) and/or shortwave (SW) parts of spectrum. The high-resolution thermal IR measurements are currently available from the GEO platform Fengyun $4 A$ operated by the China Meteorological Administration (CMA) and will be provided by Meteosat Third Generation Sounding (MTG-S) GEO mission to be launched in 2023. There are plans for several other GEO sounding missions, such as Geostationary Coastal and Air Pollution Events (GEO-CAPE) planned by NASA, Geostationary Environmental Monitoring Spectrometer (GEMS) considered by the Korea Meteorological Administration, and Infrared Fourier-Transform Spectrometer-Geostationary (IRFS-GS) under development by the Russian Federal Space Agency and the Federal Service for Hydrometeorology and Environmental Monitoring. In principle, these instruments with some adjustments can be utilized for measurements from $\mathrm{HEO}$ and $\mathrm{MEO}$ platforms, as discussed by Nassar et al. (2019). The typical refresh rate of sounding instruments to achieve the full disk (or extended spatial area) coverage is around $60 \mathrm{~min}$; that is, it is significantly longer than the refresh cycle of the imagers. Although, there are certain applications that may significantly benefit from the extended sounding capacity from the $\mathrm{HEO}$ or MEO systems, for example those requiring detailed sampling of the diurnal cycle, the $\mathrm{HEO} / \mathrm{MEO}$ sounding capacity will compete with LEO sounding capacity from current and future systems, such as Atmospheric Infrared Sounder (AIRS) on Aqua spacecraft, Infrared Atmospheric Sounding Interferometer (IASI) on board $M E T O P-A, M E T O P-B$, and $M E T O P-C$ platforms, and Cross-Track Infrared Sounder (CrIS) operated by NOAA. These LEO sounding systems can provide global sounding information several times per day (Trishchenko and Garand 2012). That said, the high temporal availability of cloud analyses from MEO or HEO in the polar regions could be used advantageously to support the assimilation of radiances from hyperspectral sounders present on LEO platforms. As well, in the overlap region with GEO imagery, that cloud analysis could be superior to the one derived from the GEO platform at high viewing angles.

From the space radiation point of view the $24-\mathrm{h} \mathrm{MEO}$ system has the most favorable environment, followed by 16-, 15-, 14-, and 12-h HEO constellations. In conclusion, continuous meteorological imaging of polar regions can be achieved from either MEO or HEO constellations. It is not obvious which approach is preferable considering the multiple factors involved, such as cost, complexity of data acquisition and processing, ionizing radiation, and continuity of operations.

Acknowledgments. The use of the ESA's Space Environment Information System (SPENVIS) (publicly accessible at https://www.spenvis.oma.be) is gratefully acknowledged. This work benefited from participation of two authors (Alexander Trishchenko and Louis Garand) in the ESA-supported project "Arctic Imager Mission Requirements Consolidation Study" (Contract 4000122460/18/NL/CT). The paper was assigned the NRCan Contribution Number 20190018.

\section{APPENDIX A}

\section{Basic Equations for Viewing Geometry and Overlapping Circles on a Sphere}

The basic chart for viewing geometry from $\mathrm{HEO}$ or GEO satellite and notations are displayed in Fig. A1. Point $\mathrm{S}$ denotes the satellite position, point $\mathrm{O}$ corresponds to the center of Earth, and $\mathrm{P}_{1}$ and $\mathrm{P}_{2}$ denote the position of subsatellite point and the intersection of line of sight with the surface. We are interested in the relation between angle $\varphi$ (or latitude span), scan angle $\beta$, and VZA $\theta$ as a function of satellite altitude $h$ and Earth's radius $R_{e}$.

The latitude span $\varphi$ can be derived as a function of the VZA (i.e., $\theta$ ) from the two equations [Eqs. (A1) and (A2)] below:

$$
\begin{aligned}
& \varphi=\theta-\beta \quad \text { and } \\
& \beta=\arcsin \left(\frac{R_{e}}{R_{e}+h} \sin \theta\right) .
\end{aligned}
$$

Figure A2 shows two overlapping circles $\left(O_{1}\right.$ and $\left.O_{2}\right)$ representing the FOVs of two satellites orbiting at the same altitude. It can be a pair of GEO or MEO satellites. The following relations hold:

$$
\begin{aligned}
& \angle \mathrm{P}_{1} \mathrm{OO}_{1}=\angle \mathrm{P}_{2} \mathrm{OO}_{2}=\varphi, \\
& \angle \mathrm{O}_{1} \mathrm{OO}_{2}=2 \delta, \\
& \angle \mathrm{O}_{1} \mathrm{OC}=\angle \mathrm{O}_{2} \mathrm{OC}=\delta, \\
& \angle \mathrm{P}_{3} \mathrm{OP}_{4}=2 \varepsilon, \quad \text { and } \\
& \angle \mathrm{P}_{3} \mathrm{OC}=\angle \mathrm{P}_{4} \mathrm{OC}=\varepsilon .
\end{aligned}
$$




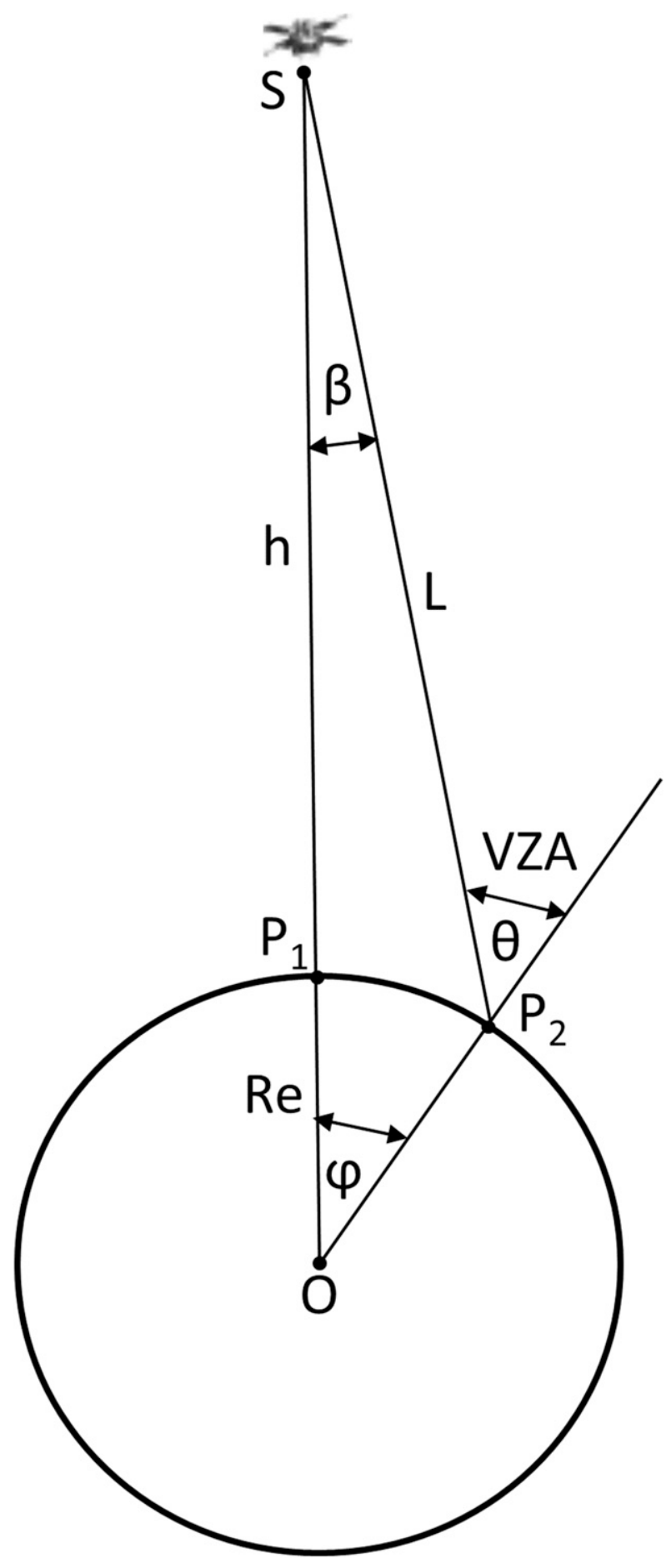

FIG. A1. Chart and notations (variables) for viewing geometry on a sphere.

Points $\mathrm{O}_{1}, \mathrm{C}$, and $\mathrm{O}_{2}$ lie along the equator for the case of GEO satellites. Point $\mathrm{C}$ denotes the North (or South) Pole for the case of MEO satellites. The angle $\varepsilon$ corresponds to latitude of intersection of two GEO FOVs shown in Figs. 1a and 1b. The angle $\varepsilon$ corresponds to the maximum colatitude (i.e., $90^{\circ}$ latitude) of continuous coverage area for the MEO constellation. The angle $2 \delta$ determines the angular distance between two satellites on a circular orbit.

In the case of two GEO satellites, we are interested to find the angle $\varepsilon$ (latitude of intersection of the FOV) as a function of satellite angular distance $\delta$ and latitude span (or FOV size) $\varphi$. Equations (A1) and (A2) express the dependence on the VZA $\theta$. For the basic equidistant sixsatellite GEO configuration along the equator, the angle $\delta=360^{\circ} / 6 / 2=30^{\circ}$. For a different number of satellites in the constellation $N$ the angle $\delta=360^{\circ} / N / 2$.

In the case of two MEO satellites, we are interested to find the angular spacing between two satellites (i.e., the angle $2 \delta$ ) corresponding to the selected minimum latitude (or maximum colatitude) of continuous coverage for a selected maximum VZA limit (or related angle $\varphi$ ). The number of satellites $N$ in the MEO constellation can be determined as

$$
N=\left\lceil 360^{\circ} /(2 \delta)\right\rceil
$$

where the \lceil\rceil operation means rounding to the smallest following integer value (ceiling function), because the number of satellites should be an integer value. For example, if value $N=3.25$ is obtained as a result of division in Eq. (A8), then four satellites are required to ensure continuous coverage.

The above angles can be computed in a straightforward way using simple analytic geometry calculations. First, we select the right-handed coordinate system as follows: the $Z$ axis is directed from point $\mathrm{O}$ to point $\mathrm{C}$, the $Y$ axis is directed from point $\mathrm{O}$ perpendicular to the $Z$ axis in the plane defined by points $\mathrm{O}_{1} \mathrm{OO}_{2}$, and the $X$ axis is directed from point $\mathrm{O}$ perpendicular to the $Z$ axis in the plane defined by points $\mathrm{P}_{3} \mathrm{OP}_{4}$. We introduce $(x, y, z)$ components of the following vectors:

$$
\begin{aligned}
\mathbf{C O} & =\left\{x_{1}, y_{1}, z_{1}\right\}, \\
\mathbf{C P}_{3} & =\left\{x_{2}, y_{2}, z_{2}\right\}, \quad \text { and } \\
\mathbf{O}_{\mathbf{1}} \mathbf{P}_{3} & =\left\{x_{3}, y_{3}, z_{3}\right\},
\end{aligned}
$$

where

$$
\begin{aligned}
& x_{1}=0, \quad y_{1}=-R_{e} \sin \delta, \quad z_{1}=R_{e}(\cos \delta-1), \\
& x_{2}=R_{e} \sin \varepsilon, \quad y_{2}=0, \quad \text { and } \quad z_{2}=R_{e}(\cos \varepsilon-1) .
\end{aligned}
$$

From vector geometry, it follows that

$$
\mathbf{O}_{1} \mathbf{P}_{3}=\mathrm{CP}_{3}-\mathrm{CO}_{1}
$$




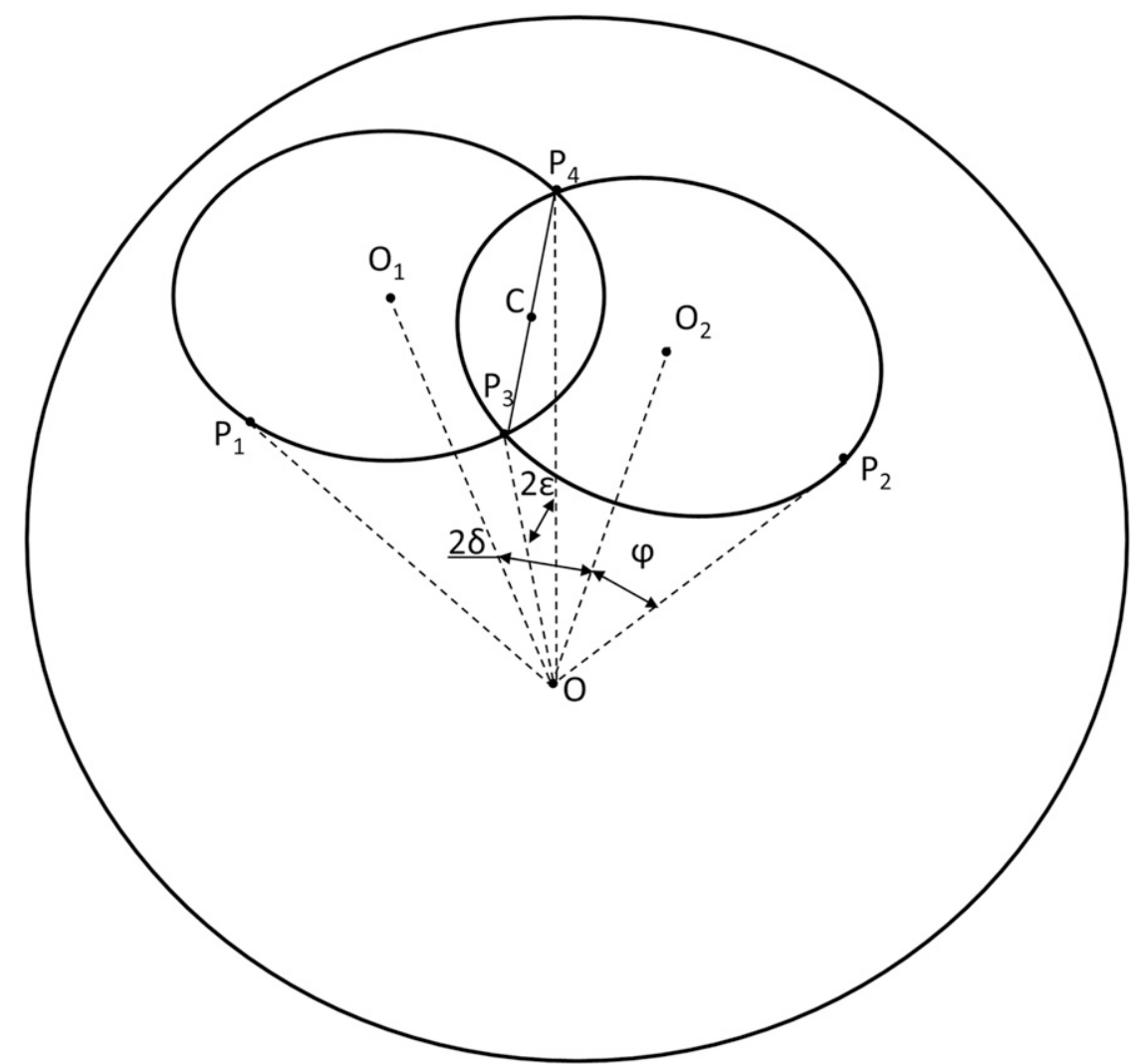

FIG. A2. Geometry and notations (variables) for the overlapping circles on a sphere.

then

$$
\begin{aligned}
& x_{3}=x_{2}-x_{1}=R_{e} \sin \varepsilon, \\
& y_{3}=y_{2}-y_{1}=R_{e} \sin \delta, \quad \text { and } \\
& z_{3}=z_{2}-z_{1}=R_{e}(\cos \varepsilon-\cos \delta) .
\end{aligned}
$$

One can compute the module of vector $\mathbf{O}_{\mathbf{1}} \mathbf{P}_{\mathbf{3}}$ equal to $R_{3}$ as

$$
\begin{aligned}
R_{3}^{2} & =x_{3}^{2}+y_{3}^{2}+z_{3}^{3}=R_{e}^{2}\left[\sin ^{2} \varepsilon+\sin ^{2} \delta+(\cos \varepsilon-\cos \delta)^{2}\right] \\
& =2 R_{e}^{2}(1-\cos \delta \cos \varepsilon) \\
R_{3} & =\sqrt{2} R_{e}(1-\cos \delta \cos \varepsilon)^{0.5} .
\end{aligned}
$$

At the same time $R_{3}$ can be derived from the Pythagoras theorem as follows:

$$
R_{3}=2 R_{e} \sin (\varphi / 2)
$$

Equating Eqs. (A11) and (A12) and recalling that $\cos 2 x=\cos ^{2} x-\sin ^{2} x$ leads to the relationship between angles $\delta, \varepsilon$, and $\varphi$ :

$$
\cos \varphi=\cos \delta \cos \varepsilon .
$$

The condition of overlap should be fulfilled, which leads to

$$
\delta \leq \varphi \leq \pi / 2 .
$$

The pixel growth factor $F_{\mathrm{PGF}}$ as a function of VZA can be computed as

$$
F_{\mathrm{PGF}}=L /(h \cos \theta) \text {. }
$$

\section{APPENDIX B}

\section{List of Main Acronyms/Notations and Their} Definitions

ABI

AIM-North

AIRS

AMV

CMA
Advanced Baseline Imager

Atmospheric Imaging Mission for Northern Regions

Atmospheric Infrared Sounder

Atmospheric motion vector China Meteorological Administration 


\begin{tabular}{|c|c|}
\hline CrIS & Cross-Track Infrared Sounder \\
\hline ESA & European Space Agency \\
\hline EUMETSAT & $\begin{array}{l}\text { European Organisation for the Exploi- } \\
\text { tation of Meteorological Satellites }\end{array}$ \\
\hline FCI & Flexible Combined Imager \\
\hline FOV & Field of view \\
\hline GEMS & $\begin{array}{l}\text { Geostationary Environmental Moni- } \\
\text { toring Spectrometer }\end{array}$ \\
\hline GEO & Geostationary orbit \\
\hline GEO-CAPE & $\begin{array}{l}\text { Geostationary Coastal and Air Pollu- } \\
\text { tion Events }\end{array}$ \\
\hline HEO & Highly elliptical orbit \\
\hline IASI & $\begin{array}{l}\text { Infrared Atmospheric } \\
\text { Interferometer }\end{array}$ \\
\hline IR & Infrared \\
\hline
\end{tabular}

IRFS-GS Infrared Fourier-Transform Spectrometer-

JMA Japan Meteorological Agency

KMA Korea Meteorological Administration

LEO

MAA $(\nu)$

MAP

MEO

Meteosat

METOP

MTG

NASA

NOAA

$\operatorname{PGF}\left(F_{\mathrm{PGF}}\right)$

P1 (P3)

$\operatorname{RAAN}(\Omega)$

SPENVIS

SW

TAP

TID

TIROS

$\operatorname{VZA}(\theta)$

WMO

$a$

$e$

$h$

$i$

$N$

$R_{e}$

$\beta$

$\delta$ Geostationary

$\Phi_{\text {ap }}$

$\varphi_{\text {ap }}$

$\omega$

Low Earth orbit

Mean anomaly angle

Multiple-apogee orbit

Medium Earth orbit

Meteorological Geostationary Satellite

Meteorological Operational satellite

Meteosat Third Generation

National Aeronautics and Space Administration

National Oceanic and Atmospheric Administration

Pixel growth factor

Notation for configuration with one (three) orbital plane(s)

Right ascension of ascending node

Space Environment Information System

Shortwave

Three-apogee orbit

Total ionizing dose

Television and Infrared Observation Satellite

Viewing zenith angle

World Meteorological Organization

Semimajor axis of the orbit

Eccentricity of the orbit

Satellite altitude

Orbit inclination

Number of satellites in constellation

Earth's radius

Scan angle

Half of the angular spacing between two MEO satellites
Minimum (the southmost) latitude observed at the apogee point in the Northern Hemisphere by the HEO satellite looking across the North Pole FOV latitude span at the apogee point Argument of perigee

\section{REFERENCES}

Chobotov, V. A., 2002: Orbital Mechanics. 3rd ed. American Institute of Aeronautics and Astronautics, $460 \mathrm{pp}$.

Daisaku, U., 2016: Aerosol optical depth product derived from Himawari-8 data for Asian dust monitoring. Meteorological Satellite Center Tech. Note, 5 pp., http://www.data.jma.go.jp/ mscweb/technotes/msctechrep61-6.pdf.

Dittberner, G., S. Bajpai, B. Diedrich, L. Key, and M. Madden, 2004: Advanced technology opportunities for future environmental satellite systems. Proc. 2004 Meteorological Satellite Conf., Prague, Czech Republic, EUMETSAT.

$\longrightarrow,-$ A. J. Gerber Jr., R. Baron, and F. Rogez, 2006: A medium Earth orbit constellation for polar wind measurements. Proc. 2006 Meteorological Satellite Conf., Helsinki, Finland, EUMETSAT.

Emmanuel, A., J. Raghavan, R. Harris, and P. Ferguson, 2014: A comparison of radiation shielding effectiveness of materials for highly elliptical orbits. Adv. Space Res., 53, 1143-1152, https://doi.org/10.1016/j.asr.2013.12.039.

EUMETSAT, 2011: MTG-FCI: Meteorological product requirements. EUMETSAT Tech. Doc. EUM/MTG/DOC/11/0723, $18 \mathrm{pp}$.

Garand, L., J. Feng, S. Heilliette, Y. Rochon, and A. P. Trishchenko, 2013: Assimilation of circumpolar wind vectors derived from highly elliptical orbit imagery: Impact assessment based on observing system simulation experiments. J. Appl. Meteor. Climatol., 52, 1891-1908, https://doi.org/10.1175/JAMC-D12-0333.1.

Gerber, A. J., D. M. Tralli, and S. N. Bajpai, 2005: Medium Earth orbit (MEO) as an operational observation venue for NOAA's post GOES-R environmental satellites. Proc. SPIE, 5659, https://doi.org/10.1117/12.577705.

Hernandez-Carrascal, A., and N. Bormann, 2014: Atmospheric motion vectors from model simulations. Part II: Interpretation as spatial and vertical averages of wind and role of clouds. J. Appl. Meteor. Climatol., 53, 65-82, https://doi.org/10.1175/ JAMC-D-12-0337.1.

Heynderickx, D., B. Quaghebeur, J. Wera, E. J. Daly, and H. R. D. Evans, 2004: New radiation environment and effects models in the European Space Agency's Space Environment Information System (SPENVIS). Space Wea., 2, S10S03, https://doi.org/ 10.1029/2004SW000073.

Kazuki, S., 2017: Introduction to the Himawari-8 atmospheric motion vector algorithm. Meteorological Satellite Center Tech. Note, 5 pp., http://www.data.jma.go.jp/mscweb/technotes/ msctechrep62-4.pdf.

Kidder, S. Q., and T. H. Vonder Haar, 1990: On the use of satellites in Molniya orbits for meteorological observation of middle and high latitudes. J. Atmos. Oceanic Technol., 7, 517-522, https:// doi.org/10.1175/1520-0426(1990)007<0517:OTUOSI >2.0.CO;2.

Nassar, R., and Coauthors, 2019: The Atmospheric Imaging Mission for Northern Regions: AIM-North. Can. J. Remote Sens., https://doi.org/10.1080/07038992.2019.1643707, in press. 
NOAA, 2016: GOES-R series mission requirements document (MRD), version 3.24. NOAA Tech. Doc. 410-R-MRD-0070, 143 pp., https://www.goes-r.gov/syseng/docs/MRD.pdf.

Sawyer, D. M., and J. I. Vette, 1976: AP-8 trapped proton environment for solar maximum and solar minimum. NASA Goddard Space Flight Center Rep. NSSDC/WDCA-R\&S 76-06, 176 pp.

Schmit, T. J., M. M. Gunshor, W. P. Menzel, J. J. Gurka, J. Li, and A. S. Bachmeier, 2005: Introducing the next-generation Advanced Baseline Imager (ABI) on GOES-R. Bull. Amer. Meteor. Soc., 86, 1079-1096, https://doi.org/10.1175/BAMS86-8-1079.

, P. Griffith, M. M. Gunshor, J. M. Daniels, S. J. Goodman, and W. J. Lebair, 2017: A closer look at the ABI on the GOES-R series. Bull. Amer. Meteor. Soc., 98, 681-698, https://doi.org/ 10.1175/BAMS-D-15-00230.1.

Seltzer, S. M., 1980: SHIELDOSE: A computer code for spaceshielding radiation dose calculations. National Bureau of Standards Tech. Note 1116, 69 pp., https://nvlpubs.nist.gov/ nistpubs/Legacy/TN/nbstechnicalnote1116.pdf.

Takahito, I., and U. Daisaku, 2016: Clear sky radiance (CSR) product derived from Himawari-8 data. Meteorological Satellite Center Tech. Note, 6 pp., http://www.data.jma.go.jp/mscweb/ technotes/msctechrep61-5.pdf.

Trichtchenko, L. D., L. V. Nikitina, A. P. Trishchenko, and L. Garand, 2014: Highly elliptical orbits for Arctic observations: Assessment of ionizing radiation. Adv. Space Res., 54, 2398-2414, https://doi.org/10.1016/j.asr.2014.09.012.

Trishchenko, A. P., and L. Garand, 2011: Spatial and temporal sampling of polar regions from two-satellite system on Molniya orbit. J. Atmos. Oceanic Technol., 28, 977-992, https://doi.org/10.1175/JTECH-D-10-05013.1.

_, and —, 2012: Observing polar regions from space: Advantages of a satellite system on a highly elliptical orbit versus a constellation of low Earth polar orbiters. Can. J. Remote Sens., 38, 12-24, https://doi.org/10.5589/m12-009.

- _ _ , and L. D. Trichtchenko, 2011: Three-apogee 16-h highly elliptical orbit as optimal choice for continuous meteorological imaging of polar regions. J. Atmos. Oceanic Technol., 28, 14071422, https://doi.org/10.1175/JTECH-D-11-00048.1.

,,,--- and L. V. Nikitina, 2016: Multiple-apogee highly elliptical orbits for continuous meteorological imaging of polar regions. Bull. Amer. Meteor. Soc., 97, 19-24, https://doi.org/ 10.1175/BAMS-D-14-00251.1.

_ L. D. Trichtchenko, and L. Garand, 2019: Highly elliptical orbits for polar regions with reduced total ionizing dose. Adv. Space Res., 63, 3761-3767, https://doi.org/10.1016/j.asr.2019.04.005.

Vette, J. I., 1991: The AE-8 trapped electron model environment. NASA Space Science Data Center Rep. NSSDC/WDC-AR\&S 91-24, $138 \mathrm{pp}$

WMO, 2009. Vision for the Global Observing System (GOS) in 2025. WMO Doc., 6 pp., http://www.wmo.int/pages/prog/sat/ documents/SAT-GEN_ST-11-Vision-for-GOS-in-2025.pdf.

, 2017: Vision for the WMO Integrated Global Observing System (WIGOS) component systems in 2040: WIGOS Vision Space 2040, draft v1.1. WMO Doc., 16 pp., http://www.wmo.int/ pages/prog/sat/meetings/documents/IPET-SUP-3_INF_0601_WIGOS-Vision-Space2040-Draft1-1.pdf.

_- 2019: OSCAR: Observing Systems Capability Analysis and Review Tool. WMO, https://www.wmo-sat.info/oscar/satellites/ view/465.

Xapsos, M. A., G. P. Summers, J. L. Barth, E. G. Stassinopoulos, and E. A. Burke, 1999: Probability model for worst case solar proton event fluences. IEEE Trans. Nucl. Sci., 46, 1481-1485, https://doi.org/10.1109/23.819111.

,,,--- , and,- 2000 : Probability model for cumulative solar proton event fluences. IEEE Trans. Nucl. Sci., 47, 486-490, https://doi.org/10.1109/23.856469. 